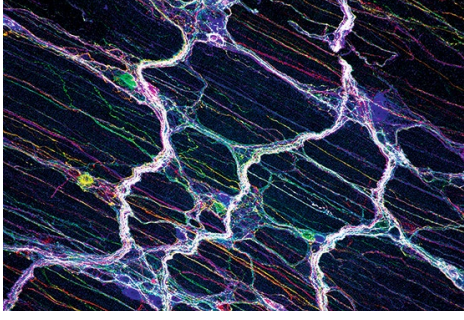

Image depicts the enteric neuronal networks of mouse small intestine tissue. Enteric neurons were labelled using adeno-associated virus vectors containing fluorescent reporters, expression of which depends on Cre recombinase. Image courtesy of Y. Obata, Francis Crick Institute, UK.

Finally, the researchers showed that wild-type mice treated with antibiotics had higher intestinal transit times and lower Ahr expression in enteric neurons than wild-type mice not treated with antibiotics. Expression of $A h r$ in the antibiotic-treated mice using an adeno-associated virus vector markedly reduced intestinal transit time.

In sum, the researchers identify AHR signalling as part of an ENS pathway that regulates gut motility in a microbiota-dependent manner. Thus, modulating AHR signalling in the ENS could have implications for the treatment and management of conditions associated with altered gut motility.

Jordan Hindson

ORIGINAL ARTICLE Obata, Y. et al. Neuronal programming by microbiota regulates intestinal physiology. Nature 578, 284-289 (2020)

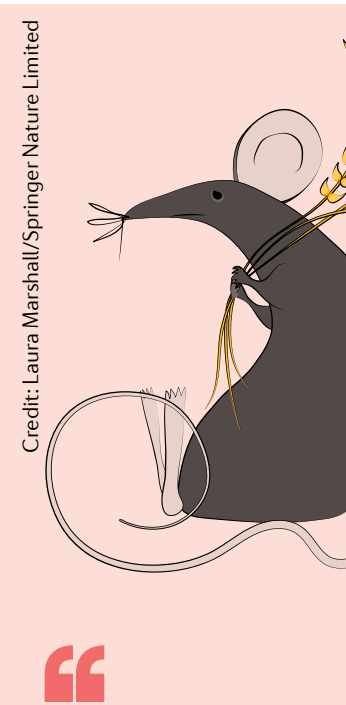

the villous architecture was restored once the mice had been fed a glutenfree diet key effector cells that mediated the tissue destruction in the small intestine of the mice. Moreover, important roles for $\mathrm{CD}^{+} \mathrm{T}$ cells, IFN $\gamma$ and transglutaminase 2 in the pathogenesis of coeliac disease were indicated in this model.

"We generated a mouse [model] based on the principle that villous atrophy will only develop when epithelial stress with IL-15 overexpression is associated with inflammatory $\mathrm{CD}^{+} \mathrm{T}$ cell responses to gluten," notes Jabri. "This new mouse model now develops villous atrophy only upon eating gluten, responds to a gluten-free diet and requires HLA-DQ8 for the development of villous atrophy," she says, adding that this mouse model can now be used to design and test novel therapeutic strategies and better understand the underlying mechanisms of the disease.

Katrina Ray

ORIGINAL ARTICLE Abadie, V. et al. IL-15, gluten and HLA-DQ8 drive tissue destruction in coeliac disease. Nature 578, 600-604 (2020)

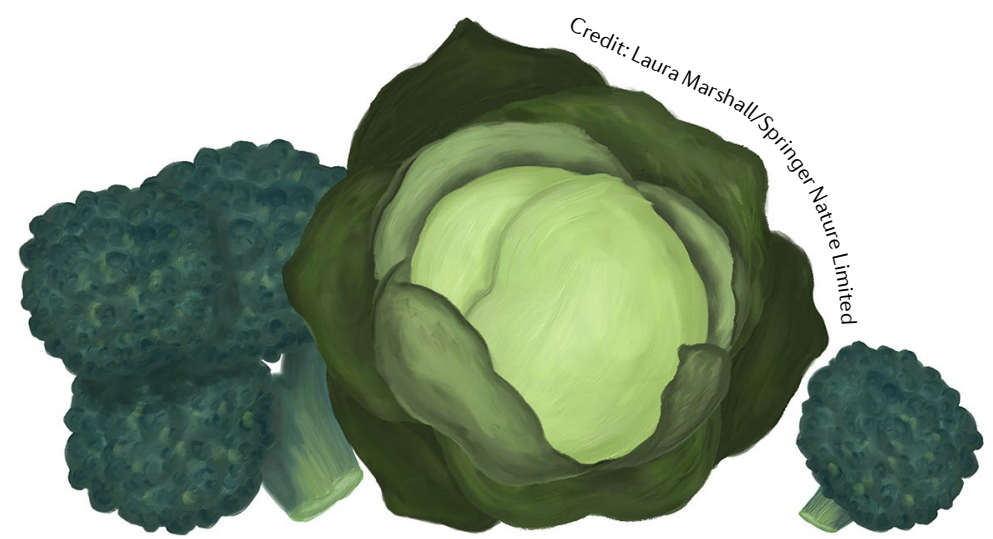

GUT MICROBIOTA

\section{Brassica vegetable metabolism by gut microbiota}

Glucosinolates (GSs) are biologically inert plant-derived metabolites that are metabolized by the gut microbiota into bioactive isothiocyanates (ITCs). GSs are abundant in Brassica vegetables such as broccoli and cabbage and have been associated with a decreased risk of several cancers owing to the chemopreventive effects of ITCs. However, the genetic mechanisms by which GSs are metabolized into ITCs in the gut were unclear. Now, in a new study, researchers have identified an operon responsible for ITC production in a key gut bacterial species.

"Previous studies by other groups have identified a number of gut bacterial isolates that are capable of metabolizing GSs to ITCs," says Elizabeth Sattely, corresponding author of the new study. To investigate the specific metabolic pathway involved in GS metabolism in Bacteroides thetaiotamicron, a prominent member of the human gut microbiota, the researchers cultured $B$. thetaiotamicron isolates in a GS-containing culture medium and then performed a genomewide transposon insertion screen. They identified BT2159-BT2156, a previously uncharacterized operon, as necessary for conversion of GSs to ITCs.

Next, expression of this operon using a plasmid-based expression system in Bacteroides fragilis, a bacterial species that does not metabolize GSs, resulted in a gain of GS-metabolizing activity in the culture medium. Then, incubation of purified BT2159-BT2156 in the presence of a GS was used to assess which of the operon-encoded proteins were necessary for GS metabolism in vitro. GS-metabolizing activity was observed when BT2158 was combined with BT2156 or BT2157.

To investigate an in vivo role for the BT2159-BT2156 operon, mice were monocolonized with $B$. thetaiotamicron with a wild-type or deleted BT2157. Mice colonized with the BT2157-deleted strain exhibited reduced gut ITC production compared with mice colonized with the wild-type strain.

Finally, the researchers examined human stool sequencing data from seven cohorts of healthy individuals, finding that the BT2160-BT2156 gene cluster was present in $>40 \%$ of all individuals in the cohorts (with the exception of one), suggesting that this operon is common in humans.

Taken together, the results identify a genetic basis for the metabolism of a dietary metabolite, both in vitro and in vivo. "Now that we have a set of genes that enable a gut bacterial strain to generate chemopreventive ITCs, we can begin to draw connections between gut microbiome composition and different health benefits that individuals might derive from the same diet," concludes Sattely. "Mechanistic insight into these gut microbial pathways that influence human health can enable the engineering of gut microbes and gut communities for therapeutic benefit."

Jordan Hindson

ORIGINAL ARTICLE Liou, C. S. et al. A metabolic pathway for activation of dietary glucosinolates by a human gut symbiont. Cell 180, 717-728 (2020) 